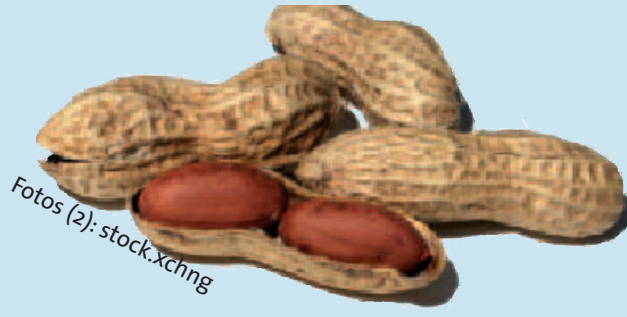

Erdnuss und Walnuss Co- oder Kreuzreaktion?

Patienten mit Erdnussallergie reagieren zu 20 bis $50 \%$ auch auf Baumnüsse wie die Walnuss. Die Aminosäuresequenzen der Allergene von Erdnuss und Walnuss weisen eine hohe Ähnlichkeit auf. Ob es sich jedoch um eine Co- oder eine Kreuzreaktivität auf Erdnuss und Walnuss handelt, war bislang unklar.

Eine Arbeitsgruppe um Dr. Kirsten Beyer, Berlin, untersuchte vor diesem Hintergrund die Bindung von spezifischen IgEAntikörpern an Sequenzepitope von Erdnuss und Walnuss bei Erdnuss- und Walnussallergikern im Vergleich zu erdnussallergischen, aber walnusstoleranten Patienten. Im Peptidmicroarray ließen sich keine Unterschiede beim Erkennen von Sequenzepitopen zwischen walnussallergischen und walnusstoleranten Erdnussallergikern finden. In zu den Sequenzepitopen der Erdnuss homologen Regionen der Walnuss fand sich nur wenig IgE-Bindung. In Inhibitionsversuchen ließen sich für die getesteten Sequenzen der Hauptallergene der Erdnuss keine relevanten peptidspezifischen IgE-Antikörper nachweisen, die mit homologen Walnusssequenzen kreuzreagieren.

Die klinisch häufige Co-Allergie gegen Erdnuss und Walnuss scheint also nicht auf kreuzreagierende IgE-Antikörper gegen homologe Sequenzepitope zurückzuführen zu sein, so das Fazit.

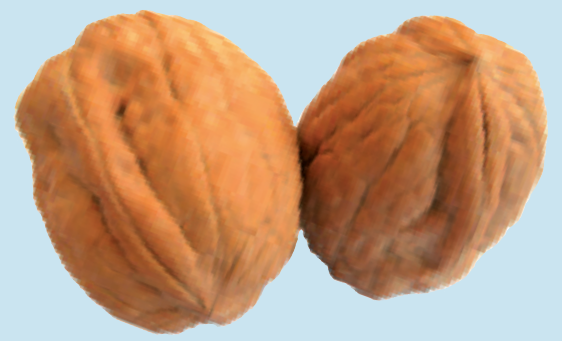

Rosenfeld L, Shreffler W, Bardina L, Sampson H, Niggemann B, Wahn U, Beyer K. Walnussallergie bei Erdnussallergikern - Häufigkeit und die Bedeutung von homologen Sequenzepitopen in der klinischen Reaktivität. 20. Mainzer Allergie-Workshop, Mainz, 7./8. März 2008

\section{Ekzemreaktion auf Nahrungsmittel?}

\section{Nicht selten beobachten Patienten mit atopischer Dermatitis, dass} sich ihr Hautzustand nach dem Verzehr bestimmter Lebensmittel verschlechtert. Bei der diagnostischen Abklärung kann der Atopie-PatchTest die orale Provokation ergänzen.
B ei Hinweisen auf eine Ekzemreaktion aufgrund einer Nahrungsmittelallergie wird bei Patienten mit atopischer Dermatitis eine orale Provokationstestung empfohlen. Damit man keine Spättypreaktionen übersieht, sollte die Haut auch am Tag nach der Provokation untersucht werden.

Um die diagnostische Wertigkeit der In-vitro-Diagnostik sowie des AtopiePatch-Tests zu evaluieren, analysierte eine Arbeitsgruppe um Dr. Annice Heratizadeh, Hannover, retrospektiv die Daten von 138 Patienten mit atopischer Dermatitis und Verdacht auf eine Allergie gegen birkenpollenassoziierte Nahrungsmittel wie Haselnuss, Karotte, Apfel oder Sellerie. 40 der 138 Patienten (29\%) zeigten in der oralen, doppelblinden und plazebokontrollierten Provokation mit birkenpollenassoziierten Nahrungsmitteln eine positive Reaktion und galten als Responder. 17 Patienten zeigten dabei eine Soforttypreaktion, 15 eine kombinierte Sofort- und Spättypreaktion sowie acht eine isolierte Ekzemreaktionen.

Bezüglich der IgE-Titer fanden sich keine signifikanten Unterschiede zwi- schen den Respondern und den Patienten, bei denen keine Allergie gegenüber birkenpollenassoziierten Nahrungsmitteln gesichert werden konnte. Bei 15 Respondern und bei 22 Non-Respondern wurde zudem ein Atopie-PatchTest mit Birkenpollenextrakt durchgeführt: $18 \%$ der Non-Responder (4/22) sowie $40 \%$ der Responder (6/15) waren positiv.

Der Atopie-Patch-Test mit Birkenpollenextrakt kann daher eine sinnvolle Ergänzung der Diagnostik einer Nahrungsmittelallergie bei Patienten mit atopischer Dermatitis darstellen, resümierte Heratizadeh. Der Goldstandard der Diagnostik ist allerdings weiterhin der doppelblinde und plazebokontrollierte Provokationtest.

abd

\section{Heratizadeh A, Killig C, Bouras A, Ottens S,} Werfel T. Birkenpollenassoziierte

Nahrungsmittelallergie bei Patienten mit atopischer Dermatitis - Häufigkeit und diagnostische Wertigkeit der In-vitroDiagnostik und des Atopie Patch Testes. 20. Mainzer Allergie-Workshop, Mainz, 7./8. März 2008

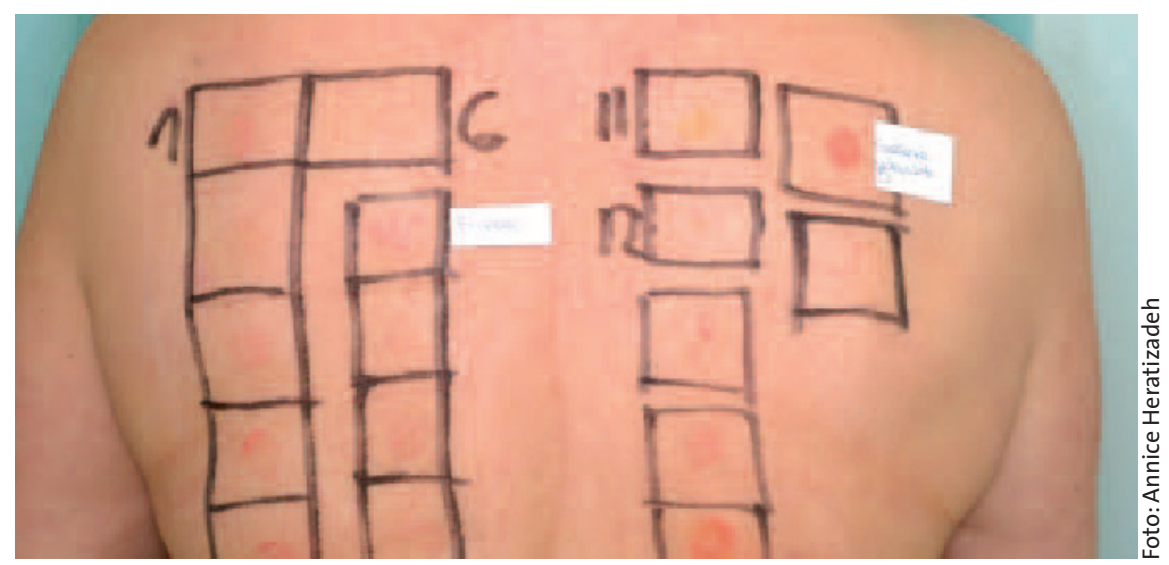

Der Atopie-Patch-Test kann bei der Diagnostik des atopischen Ekzems wertvolle Hinweise liefern. 